\title{
SURPRISE IS RESEARCH'S REPRISE
}

\author{
by Bernard Dixon
}

\section{6 \\ $\mathrm{T}$}

he science and technology of releasing genetically modified organisms into the environment, and the associated techniques of risk assessment, have now well and truly arrived," said John Lake, Director of the European Environmental Research Organization (EERO), recently at Wageningen in the Netherlands. "No longer are we working in the dark, or hazarding guesses about the possible benefits or potential risks of released GMOs. There is now a solid corpus of information upon which to make predictions and build confidence."

Lake's point is well-taken. Indeed, his argument found tangible form in that he was speaking during a two-part EERO course, spanning no less than ten days, at which participants heard a wealth of ideas about every conceivable aspect of the subject. After three days of presentations on topics such as gene transfer, self-destruct strategies, and the monitoring and enumeration of organisms in the soil, they moved to the laboratory bench for an equally intensive sequence of practical work.

Especially since ecological and social dimensions were far from neglected, this was all to the good. Had some of the opponents of deliberate release achieved their aim a few years ago, John Lake would have been deprived of his corpus of knowledge and the EERO course would have been unthinkable. The one certain consequence of a moratorium on research is that learning becomes impossible. As in the Dark Ages, everything simply grinds to a halt.

All the more telling, therefore, was the evidence provided by this highly successful occasion suggesting that we do not yet know everything that we need to know if we are to evaluate exhaustively the performance of microorganisms disseminated in the biosphere for agricultural and other purposes. When I last touched on this topic (Bio/Technology 9: 403, 1991), it was to underline implications of the continuing stream of discoveries regarding the richness of gene transfers in nature, especially those that were previously unsuspected. At the Wageningen meeting, however, there was news not of some rare and atypical variety of microbial sex but of a hitherto unknown facet of everyday bacterial behavior.

\section{SUICIOE CASSETTES}

The person who announced the new finding was Soren Molin from the Technical University of Denmark at Lyngby. He had been invited to speak on conditional lethal constructions, and indeed did so, describing several of the suicide containment systems which he and his colleagues have developed in recent years. There was particular interest in a system of this sort which they have designed to control genetically engineered microorganisms capable of degrading substituted benzoates, so that the cells die off once the pollutant has been dealt with. The system consists of two parts. One is the fusion between the promoter of the Pseudomonas putida TOL plasmid-encoded meta-cleavage pathway operon and the lacl gene coding for Lac repressor plus $x y l S$, coding for the positive regulator of the meta-cleavage operon. The other element carries a fusion between the $\mathrm{P}_{\text {tac }}$ promoter and the gef gene, which codes for a killing function.

It works like this. In the presence of $x y l S$ effectors, Lac1 protein is synthesised. This prevents expression of the killing capacity. But in the absence of effectors, expression of the $P_{\text {tac }}:: g e f$ cassette is no longer held in check, and a high rate of killing thus occurs. By substituting $x y l S$ with another mutant regulator with altered effector specificity and higher affinity for benzoates, populations able to degrade a wider range of benzoates at micromolar substrate concentrations can be controlled. Although a small proportion of engineered organisms become resistant to Gef, Molin is confident that this system will have valuable applications in microbial communities able to metabolise environmental pollutants ranging from methyl- and methoxybenzoates to methyland chlorosalicylates.

\section{E. COLI CAUSES A STIR}

But it was something in principle much less sophisticated that caused the major stir in the EERO audience. En route to Wageningen, Molin had been reading the thesis for a Ph.D. awarded recently to Ritta Mikkola at Uppsala University in Sweden. Working under C.G. Kurland, Mikkola has been comparing two basic attributes of laboratory strains of Escherichia coli with the same organism recovered from patients. Firstly, she looked at growth rate and discovered, to everyone's surprise, that the clinical isolates grew more slowly than the lab varieties. Next, she examined their ribosome efficiency and found the same thing. Contrary to previous belief that bacteria in the highly competitive world "out there" are the more efficient, strains grown in artificial media for many years came out top on both measures.

When the various isolates were put on to starvation media as a test of their viability, it was the faster growing cells that were the first to die. Cultured in a chemostat, however, all of the strains eventually attained the same growth rate (and ribosome efficiency) - this being achieved by only one to three mutations over 300 generations.

So once again here is something rather remarkable which we did not know hitherto, and which has undoubted relevance to the assessment of the fate of released organisms. Mikkola and Kurland's work shows that in nature there can be selection for slow growth-rather than the fast growth predicted on the basis of previous "knowledge."

Soren Molin has a neat analogy for the risk assessment of GMOs. Imagine two youngsters standing on one side of a highway with a candy store opposite. One child sees the shop and instinctively rushes into the roadway, endangering his life and that of passing motorists too. The other child waits and gradually discerns the two-way flow of vehicles, the variations in flow and the gaps resulting from intersections and controlling lights. Only when he fully understands the rules of the traffic does he make his move-safely.

Do we yet understand the rules of traffic? 